PROCEEDINGS OF THE AMERICAN MATHEMATICAL SOCIETY

Volume 124, Number 8, August 1996

\title{
SPECTRALLY DETERMINED GROWTH IS GENERIC
}

\author{
MICHAEL RENARDY \\ (Communicated by Palle E. T. Jorgensen)
}

\begin{abstract}
Let $A$ be the infinitesimal generator of a $C_{0}$-semigroup of operators in a Hilbert space. We consider the class of operators $A+B$, where $B$ is bounded. It is proved that the spectrum of $A+B$ determines the growth of the associated semigroup for "most" operators $B$ (in the sense of Baire category).
\end{abstract}

\section{INTRODUCTION}

As is well known, the stability of the system $\dot{y}=A y$ of linear ODEs with constant coefficients is determined by the eigenvalues of the matrix $A$. In infinite dimensions, the issue is more complex. The appropriate abstract setting is that of $C_{0}$-semigroups of linear operators in a Banach space $X$. Let $A$ be an infinitesimal generator, and let $\exp (A t)$ be the associated semigroup. As usual, we define the type of the semigroup by

$$
\omega(A)=\lim _{t \rightarrow \infty} \log \left\|e^{A t}\right\| / t,
$$

and we define the spectral bound as

$$
r(A)=\sup \{\operatorname{Re} \lambda \mid \lambda \in \sigma(A)\},
$$

where $\sigma(A)$ denotes the spectrum of $A$.

It is well known that $\omega(A) \geq r(A)$. However, equality is not true in general. A first counterexample was given in [1]. Zabczyk [4] has given a much more natural example, and his ideas were recently used to show that counterexamples can be as simple and natural as a lower order perturbation of the wave equation [3]. This, in particular, destroys all hopes that "applied" problems are somehow well-behaved enough to avoid "pathological" cases where $\omega(A)$ is strictly greater than $r(A)$. This is disturbing, since computation of the spectrum is the most widely used practical method for assessing the stability of physical systems.

In this note, we take a different view. Rather than considering one operator $A$, we shall look at the class of operators $A+B$, where $B$ ranges over the set $B(X)$ of all bounded linear operators in $X$. Let us define

$$
S(A)=\{B \in B(X) \mid \omega(A+B)>r(A+B)\} .
$$

We shall prove the following result.

Received by the editors January 9, 1995 and, in revised form, February 23, 1995.

1991 Mathematics Subject Classification. Primary 47D06.

Key words and phrases. Linear stability, semigroups of operators.

This research was supported by the National Science Foundation under Grant DMS-9306635 and by the Office of Naval Research under Grant N00014-92-J-1664.

(C)1996 American Mathematical Society 
Theorem. If $X$ is a Hilbert space, then the set $S(A)$ is of first category in $B(X)$ (with the topology of the operator norm).

In a sense this theorem asserts that the spectrum determines the growth of the semigroup "most of the time".

\section{Proof of the TheOrem}

To prove the theorem, we shall first establish a few lemmas.

Lemma 1. Let $C$ be a closed operator in $X$ with bounded inverse. Assume that $\left\|C^{-1}\right\|>1 / \epsilon$. Then there exists $D \in B(X)$ with $\|D\| \leq 2 \epsilon$ such that 0 is a simple isolated eigenvalue of $C+D$.

Proof. It follows from the assumptions that there exists $x_{0} \in D(C)$ such that $\left\|x_{0}\right\|=1$ and $\left\|C x_{0}\right\| \leq \epsilon$. By the Hahn-Banach theorem we can pick $y_{0} \in X^{*}$ such that $\left\|y_{0}\right\|=1$ and $\left(y_{0}, x_{0}\right)=1$. By choosing $y_{1}$ to be a small perturbation of $y_{0}$, we can arrange it so that $\left\|y_{1}\right\| \leq 3 / 2,\left(y_{1}, x_{0}\right)=1$ and $\left(y_{1}, C^{-1} x_{0}\right) \neq 0$. Let us now define

$$
D_{1} x=-\left(y_{1}, x\right) C x_{0} .
$$

Then $\left\|D_{1}\right\| \leq \epsilon\left\|y_{1}\right\| \leq 3 \epsilon / 2$. Since $D_{1}$ has finite rank, $C+D_{1}$ has Fredholm index zero. Moreover,

$$
\left(C+D_{1}\right) x_{0}=0,\left(y_{1}, C^{-1}\left(C+D_{1}\right) x\right)=0 .
$$

Let us define the subspace

$$
X_{1}=\left\{x \in X \mid\left(y_{1}, C^{-1} x\right)=0\right\} .
$$

Since $X_{1}$ is the nullspace of a linear functional, it has codimension one. We can decompose $X=X_{1}+\left[x_{0}\right]$, where $\left[x_{0}\right]$ denotes the linear span of $x_{0}$. One easily checks that this decomposition is also continuous in $D(C)$ (equipped with the usual graph norm). Now consider the restriction of $C+D_{1}$ to $D(C) \cap X_{1}$, viewed as an operator in $X_{1}$. Since $\left(C+D_{1}\right) x_{0}=0$, the range of the restriction is the same as the range of $C+D_{1}$, but changing the space from $X$ to $X_{1}$ has reduced the codimension by 1 . Since $x_{0}$ is in the nullspace of $C+D_{1}$, the restriction also reduces the dimension of the nullspace by one. Hence the restricted operator still has Fredholm index zero, and hence there exists an arbitrarily small perturbation $D_{2} \in B\left(X_{1}\right)$ such that $C+D_{1}+D_{2}$ is invertible on $X_{1}$. We extend $D_{2}$ to all of $X$ by setting $D_{2} x_{0}=0$. The operator $D=D_{1}+D_{2}$ is the one we seek.

We now define $Q(A, \epsilon)$ to be the set of all $B \in B(X)$ such that $A+B$ has a simple isolated eigenvalue with real part greater than $\omega(A+B)-\epsilon$.

Lemma 2. $Q(A, \epsilon)$ is open.

Proof. Let $B \in Q(A, \epsilon)$. Then there exists $\delta>0$ such that $A+B$ has a simple isolated eigenvalue with real part greater than $\omega(A+B)-\epsilon+\delta$. If $\left\|B_{1}\right\|$ is small enough, then $\omega\left(A+B+B_{1}\right) \leq \omega(A+B)+\delta / 2$, and $A+B+B_{1}$ has a simple isolated eigenvalue within distance $\delta / 2$ of the isolated eigenvalue of $A+B$. This implies that $A+B+B_{1} \in Q(A, \epsilon)$, and hence $Q(A, \epsilon)$ is open.

Lemma 3. Assume that $X$ is a Hilbert space. Then $Q(A, \epsilon)$ is dense in $B(X)$ with the norm topology. 
Proof. Let $B \in B(X)$ and $\delta>0$ be given. By a theorem of Prüß [2], the resolvent of $A+B$ is unbounded in the halfplane $\operatorname{Re} \lambda>\omega(A+B)-\delta$. Moreover, there exists a constant $M$ such that $\|\exp ((A+B) t)\| \leq M \exp ((\omega(A+B)+\delta) t)$. Now prescribe an arbitrary $\epsilon>0$. Then by Lemma 1 , there exists a perturbation $B_{1}$, with norm less than $\epsilon$, such that $A+B+B_{1}$ has an isolated simple eigenvalue in the halfplane $\operatorname{Re} \lambda>\omega(A+B)-\delta$. Moreover, the type of $A+B+B_{1}$ is at most $\omega(A+B)+\delta+M \epsilon$. Thus $B+B_{1} \in Q(A, 2 \delta+M \epsilon)$. Since $\epsilon$ was arbitrary, we can choose it such that $M \epsilon<\delta$, so that $B+B_{1} \in Q(A, 3 \delta)$.

The theorem now follows by observing that $S(A)$ lies in the complement of

$$
\bigcap_{n=1}^{\infty} Q\left(A, \frac{1}{n}\right)
$$

\section{REFERENCES}

1. E. Hille and R. S. Phillips, Functional analysis and semigroups, American Mathematical Society, Providence, 1957. MR 19:664

2. J. Prüß, On the spectrum of $C_{0}$-semigroups, Trans. Amer. Math. Soc. 284 (1984), 847-857. MR 85f: 47044

3. M. Renardy, On the linear stability of hyperbolic PDEs and viscoelastic flows, Z. Angew. Math. Phys. 45 (1994), 854-865. MR 95i:35195

4. J. Zabczyk, A note on $C_{0}$-semigroups, Bull. Acad. Polon. Sci., Ser. Sci. Math. 23 (1975), 895-898. MR 52:4025

Department of Mathematics, Virginia Tech, Blacksburg, Virginia 24061-0123

E-mail address: renardym@math.vt.edu 